\title{
How easy is it to judge ease of learning?
}

\author{
EUGENE B. ZECHMEISTER and DAVID J. BENNETT \\ Loyola University, Chicago, Illinois
}

\begin{abstract}
In two experiments, we asked college students to judge the ease of learning word pairs known to differ reliably in actual ease of learning. The independent variable in both experiments was the rate of presentation (fast and slow) of to-be-judged items. The results of both experiments revealed only small differences in decision accuracy as a function of presentation rate. This suggests that metacognitive judgments of this kind rely on rather fundamental cognitive processes, which in our view are similar to those used to discover meaning in everyday verbal messages.
\end{abstract}

Consider the following metacognitive task related to associative learning: A subject is presented a list of pairs of items (e.g., words), with the instruction to judge the ease of learning an association between members of each pair. No learning is requested of the subject; rather, the subject simply predicts (e.g., via a rating scale) how easy it would be to learn an association between members of each pair if learning were requested. These so-called ease of learning (EL) judgments reliably predict associative learning (see, e.g., Arbuckle \& Cuddy, 1969; Kearney $\&$ Zechmeister, 1989), and thus they presumably provide information on the basis of which a learner can plan efficient study. For example, Nelson and Leonesio (1988) showed that more self-paced study time was allocated to items previously identified as more difficult on the basis of EL judgments.

Kearney and Zechmeister (1989) speculated that the ability to make EL judgments would be related to associative learning ability. They found, however, only a slight relationship between performance on an associative learning task and performance on an EL task, although both tasks involved the same kind of items. Such a finding seems paradoxical, if it is assumed that effective associative learning is related to efficient use of study time based on judgments of item difficulty. A dissociation between performance on these tasks is also unexpected when viewed in terms of some interpretations of the associative learning process. Wang (1983), for example, suggests that good learners generate more effective elaborators than do poor learners. A dissociation between learning and EL measures would imply that the ability to produce effective elaborators is independent of the ability to recognize the items that can be most effectively elaborated.

\footnotetext{
We wish to acknowledge support provided by a small grant from the Loyola University Research Services department. Thanks are also due John J. Shaughnessy for his critical reading of a draft of this manuscript and James W. Hall for many helpful discussions on this topic. Requests for reprints should be addressed to Eugene B. Zechmeister, Psychology Department, Loyola University of Chicago, 6525 N. Sheridan Rd., Chicago, IL 60626.
}

In the present study, we report the results of two experiments in which we asked college students to make EL judgments of word pairs known to differ reliably in learning difficulty. The independent variable was the rate of presentation of to-be-judged items. To the extent that EL judgments "take time" to make, as might be the case if a learner must search for an effective elaborator in order to gauge pair difficulty, then EL judgments should be more accurate under slow than under fast presentation conditions. If accurate EL judgments can be made relatively quickly and efficiently, as might be the case if some simpler, even fundamental, process is involved, then the rate of presentation (within reasonable limits) should have little or no effect on accuracy. In Experiment 1, we used a forced-choice procedure; in Experiment 2, we used an absolute judgment task.

\section{EXPERIMENT 1}

\section{Method}

Subjects. The participants were 52 students from two upper-division psychology classes, who participated at the request of their respective instructors. The rationale and results of the experiment were later presented to the classes as part of a teaching unit on metacognition. Subjects were randomly assigned to the fast and slow presentation conditions and tested in small groups with materials presented in test booklets.

Materials. To-be-judged items were obtained from a list of 200 word pairs prepared by Underwood (1982). He randomly paired 400 fiveletter words from the Thorndike-Lorge (1944) word book and obtained measures of associative learning and reliability for the 200 pairs. Reliable differences in item difficulty were found, and Underwood reported a mean learning score and rank for the $\mathbf{2 0 0}$ items based on actual ease of learning. Kearney and Zechmeister (1989) showed that item learning by students in the Underwood study was highly predictive of item learning in another college population. We selected 60 of Underwood's word pairs: the 30 easiest to learn and the 30 hardest to learn. Examples of easy-to-learn word pairs are kappa-fussy, erect-floor, and totem-wives; hard-to-learn pairs are rouse-creel, taker-clomb, and firth-leach. The 30 easy and 30 hard pairs were randomly paired with the restriction that easy and hard pairs appear equally (15 times) on the left and right in the set of 30 pairs of pairs. Pairs were typed, with a space between lines, in a column on a sheet of standard paper. An additional 10 word pairs from the Underwood list (the next 5 easiest and next 5 hardest) were randomly paired and used for a practice list.

Procedure. Each subject was given a three-page test booklet and a blank sheet of paper that served as a cover sheet. The first page contained extensive task instructions, including an introduction to the EL 
task, an explanation of the kinds of items that would be judged, and examples of common forms of associative learning (e.g., learning names of state capitals). The students were told that they would see a list of pairs of word pairs and that research had shown that the two pairs in each set differed in learning difficulty. Half the students in each presentation group were asked to identify (by underlining) the easy (for people in general) word pair in each set; the other half were asked to identify the hard word pair in each set of pairs. The instructions also told the subjects to use the cover sheet to help them focus on one pair of items at a time and not to move to the next pair until they heard a tone. The subjects in the fast presentation group heard a recorded tone at 4-sec intervals; the subjects in the slow presentation group heard a tone at 12 -sec intervals. Pilot testing had shown that $4 \mathrm{sec}$ was sufficient time for subjects to read both word pairs in a set and make an EL decision, if they worked quickly and efficiently. Before the actual test, the subjects practiced the task, using a small set of items found on the second page of the booklet. If there were questions following the practice task, these were answered prior to asking the subjects to make decisions about the 30 critical pairs of items that appeared on the third page of the booklet.

\section{RESULTS AND DISCUSSION}

The mean number of correct EL decisions made by subjects in the 4-sec condition was $22.80(S D=2.85)$; the mean correct in the 12-sec condition was greater, 24.38 $(S D=1.86)$. This difference was significant (all inferences are made with alpha set at .05) $[F(1,48)=5.64$, $\left.M S_{\mathrm{e}}=5.73\right]$. Whether the subjects identified the easy or hard member of a pair was not significant as a main effect, nor did it interact with the rate variable. An examination of the number of errors made on each of the 30 critical sets revealed that the difficulty of EL discrimination correlated .83 between the two presentation rate conditions.

Although EL decisions were more accurate at the slower rate, the mean difference in accuracy was only 1.58 items (less than 10\%). Moreover, the subjects performed significantly above chance (15) even at the fast rate. Thus, comparative EL decisions are somewhat more accurate when made at a slow rate, but, at least for these materials, substantial accuracy of EL decisions was possible when discriminations had to be made very quickly. In Experiment 2 , we looked for an effect of rate of presentation when decisions were made on an absolute rather than a relative basis.

\section{EXPERIMENT 2}

\section{Method}

Subjects. The subjects were 40 volunteers from an introductory psychology class, who earned course credit for participation. These subjects were tested in pairs, using two Apple II computers located in a small testing room.

Materials. The 60 critical word pairs used in Experiment 1 appeared in the critical list of Experiment 2 . An additional 6 items ( 3 relatively easy and 3 hard) were used in a practice list ( 4 items) or as a primacy buffer ( 2 items) in the experimental list. The 60 critical word pairs were randomly ordered to produce two different forms of the list; in each case, the restriction was made that an equal number of easy and hard pairs (15) appear in each half of the test list. The primacy buffer remained the same for the two orders, which were used equally often by subjects in each presentation condition; thus, the list contained 62 pairs.

Procedure. The instructions given in Experiment 1 were modified in order to be appropriate for the absolute judgment procedure used in Experiment 2. Each subject was provided with an answer sheet numbered 1-62. Next to each number were the letters $E$ and H. The sub- jects were seated in front of a computer monitor and told that a series of word pairs would be shown on the screen in front of them. They were told that when an item was presented, they were to decide whether it would be easy $(\mathrm{E})$ or hard $(\mathrm{H})$ to learn and to indicate their choice by circling the appropriate letter on the answer sheet. The experimenter indicated that about half the items would be easy items and that half should be considered hard. Items appeared at either 4-sec or 8-sec intervals. Pilot testing had revealed that $4 \mathrm{sec}$ was enough time for subjects to read the pair and to make a response on the answer sheet, which was attached to a clipboard held by the subject. While this is the same rate as that used in Experiment 1 when two pairs had to be read, the subjects in Experiment 2, unlike those in Experiment 1, had to look away from the stimulus materials in order to indicate their EL decisions. Eight seconds were used for the slow rate (rather than $12 \mathrm{sec}$, as in Experiment 1), because the same pilot testing showed that subjects became rather impatient when pairs were presented at intervals much greater than this.

\section{RESULTS AND DISCUSSION}

The significant advantage for the slower rate obtained in Experiment 1 was not found in Experiment 2. The overall mean of the correct identifications made by the subjects $(n=20)$ in the 8 -sec presentation group was 41.3 $(S D=4.85)$; mean correct in the 4-sec group $(n=20)$ was $39.7(S D=4.86)$. The obtained $t(38)$ of 1.04 was not significant. An analysis based on $d^{\prime}$ scores also revealed no significant difference between the presentation rate groups $(t=1.19)$. The accuracy of identification of easy and hard items was similar in each group. Under a fast rate, mean hits were 20.3 and 19.4 for easy and hard items, respectively; the same relative comparison yielded means of 19.8 and 21.5 in the slow group. The subjects in the fast group overall said "easy" for .51 of the items; the subjects in the slow group responded "easy" for .47 of the 60 items.

\section{GENERAL DISCUSSION}

In the two experiments, we investigated how "easy" it is to judge ease of learning when the to-be-judged material differs reliably in actual ease of learning. The judgment task was made easy or hard by manipulating the rate at which EL decisions had to be made. The assumption was that if EL judgments are relatively difficult to make, then EL accuracy would be substantially better under slow than under fast rates of presentation. We determined presentation rates through pilot work, such that a fast rate allowed subjects just enough time to read the critical items and indicate manually an EL decision. Slow rates permitted the subjects considerably more time to consider characteristics of critical items prior to making an EL decision. When the subjects performed a forcedchoice task, there was a small, yet significant, advantage with the slow rate; when the subjects performed an absolute judgment task, there was again a small, but this time nonsignificant, advantage with the slow rate. These findings indicate that decisions about the difficulty of learning an association between members of a word pair can be made accurately in a relatively brief period of time.

There is, of course, some slippage in the process by which we make EL decisions. In neither experiment did any subject even at the slower rates perform perfectly on the EL task. (In Experiment 1, 1 subject did correctly discriminate 29 out of 30 items.) Under the slow rate of Experiment 1 , when given a forced-choice task, the subjects discriminated correctly about $80 \%$ of the time. Although differences in actual learning difficulty with the to-be-judged pairs were quite substantial, it is clear that the subjects sometimes found it hard to decide whether a pair would be easy or hard to learn. Discriminations are likely affected by individual differences in familiarity or experience with the items to be judged and possibly in understanding the nature of the projected learn- 
ing task (e.g., associative learning). We do not know, for instance, whether at times an item that is normatively "hard" would actually be easier to learn for a particular subject than the "easy" item with which it was paired. It is also possible that subjects have difficulty predicting learning ease for someone else-that is, for a "group of people," as was required in the present task.

The present results also do not identify the basis for EL decisions. Underwood (1982), after performing several correlational analyses of the items used in the present study, commented that he could not identify the critical factors that determined item difficulty. Kearney and Zechmeister (1989) did, however, show that ratings of "how easy it would be to link the members of the pair in a meaningful way" predicted item difficulty as well as did EL judgments. It seems likely that learners respond to "meaning" of the to-be-judged items. If one-or both-of the pair members is seen as meaningful, or, especially, if the relationship between pair members is judged to be meaningful, then learning is likely to judged to be easier than if little or no meaning is found. Begg, Duft, Lalonde, Melnick, and Sanvito (1989) have recently suggested that the ease of processing during study is used to predict the outcome of a future memory test. The ease of processing is affected by variations in ease of imagining, understanding, and pronouncing verbal items. We assume that when subjects are asked to predict the outcome of a future memory test before study, such factors also play a role (see also Lippman \& Kintz, 1968).

The finding of meaning in verbal messages is something that we practice every day and thus is ably accomplished by most educated adults. Gerrig (1989) points out that in everyday conversation we often need to create new meanings for old words. This sense creation is exemplified by our ability to understand metaphors and other innovative uses of words (e.g., "uniforms" for "uniformed police officers"). Both sense selection (selecting among the possible senses of a word) and sense creation rely on the comprehender's general knowledge as well as knowledge shared by specific subsets of the population (Gerrig, 1989). If we can quickly and easily make sense of a new meaning for old words, it seems that we can just as easily decide that totem-wives makes more sense than does rouse-creel. We believe that this process of making sense of verbal items provides clues to the ease with which they can be learned. People, in other words, are "meaning detectors," and the detection of meaning provides a basis for deciding what will or will not be easy to learn. This detection appears to be relatively immediate and based on reliable differences in language experiences for different items. In another context, Keppel and Zubrzycki (1977) describe associative learning as the transfer of past knowledge to the learning situation. Apparently, adult learners are able to discriminate quickly and efficiently when that transfer has taken place.

\section{REFERENCES}

Arbuckle, T. Y., \& CUdDY, L. (1969). Discrimination of item strength at time of presentation. Journal of Experimental Psychology, 81, 126-131.

BegG, I., Duft, S., Lalonde, P., Melnick, R., \& Sanvito, J. (1989). Memory predictions are based on ease of processing. Journal of Memory \& Language, 28, 610-632.

GerRIG, R. J. (1989). The time course of sense creation. Memory \& Cognition, 17, 194-207.

Kearney, E. M., \& Zechmeister, E. B. (1989). Judgments of item difficulty by good and poor associative learners. American Journal of Psychology, 102, 365-383.

KePPEL, G., \&UBRZYCKI, C. R. (1977). Selective learning and forgetting. In I. M. Birnbaum \& E. S. Parker (Eds.), Alcohol and human memory (pp. 73-95). Hillsdale, NJ: Erlbaum.

LippMan, L. G., \& KinTZ, B. L. (1968). Group predictions of item differences of CVC trigrams. Psychonomic Science, 12, 265-266.

Nelson, T. O., \& Leonesio, R. J. (1988). Allocation of self-paced study time and the "labor-in-vain effect." Journal of Experimental Psychology: Learning, Memory, \& Cognition, 14, 676-686.

THORNDIKE, E. L., \& LORGE, I. (1944). The teacher's word book of 30,000 words. New York: Bureau of Publications, Teachers College, Columbia University.

UNDERWOOD, B. J. (1982). Paired associate learning: Data on pair difficulty and variables that influence difficulty. Memory \& Cognition, 10, 610-617.

WANG, A. Y. (1983). Individual differences in learning speed. Journal of Experimental Psychology: Learning, Memory, \& Cognition, 9. 300-311.

(Manuscript received June 14, 1990.) 